\title{
The Maturation of Task Set-Related Activation Supports Late Developmental Improvements in Inhibitory Control
}

\author{
Katerina Velanova, ${ }^{1}$ Mark E. Wheeler, ${ }^{2}$ and Beatriz Luna ${ }^{1,2}$ \\ Departments of ${ }^{1}$ Psychiatry and 2 Psychology, University of Pittsburgh, Pittsburgh, Pennsylvania 15213
}

The ability to voluntarily inhibit a single response is evident early in development, even as the ability to maintain an inhibitory "task set" continues to improve. To date, functional neuroimaging studies have detailed developmental changes in systems supporting inhibitory control exerted at the single-trial level, but changes underlying the ability to maintain an inhibitory task set remain little understood. Here we present findings from a functional magnetic resonance imaging study that characterizes the development of systems supporting both transient (trial-related) and sustained (task set-related) activation during performance of the antisaccade task-an oculomotor test of inhibitory control (Hallett, 1978). Transient activation decreased from childhood to adolescence in regions known to support inhibitory processes and oculomotor control, likely reflecting less effortful response production. In contrast, sustained activation increased to adulthood in regions implicated in control. Our results suggest that development of the ability to maintain a task set is primary to the maturation of inhibitory control and, furthermore, that this ability is still immature in adolescence.

\section{Introduction}

The ability to exert inhibitory control during a single trial exists even in infancy, suggesting that the circuitry supporting transient inhibitory control is available early in development (Diamond and Goldman-Rakic, 1989; Johnson, 1995). However, the ability to exert inhibitory control in a persistent manner continues to mature through adolescence, evidenced by improved rates of correct inhibitory responding (Bedard et al., 2002). One factor that may account for this improvement is development of the ability to maintain an inhibitory "task set" over time.

A task set refers to a configuration of cognitive processes, initiated by implicit or explicit instructional cues, that is actively maintained during task performance (Monsell, 1996). Task sets are thought to play a supervisory control function, determining and guiding operations performed on transiently occurring stimuli (Dosenbach et al., 2006). In broad lay terms, a task set represents the overarching "rules" that govern task performance.

Evidence that task sets are neurally represented comes from numerous sources. However, most research has emphasized task set implementation and anticipatory neural signaling before presentation of task-relevant stimuli, as with cueing (Corbetta and Shulman, 2002) and task-switching paradigms (Sakai, 2008). In contrast, we regard signaling maintained throughout periods of task performance-the background against which transient modulation occurs_-as reflecting maintenance of a task set [sim-

Received April 1, 2009; revised July 16, 2009; accepted Sept. 2, 2009.

This work was supported by National Institute of Mental Health Grant R01 MH067924. We thank Mark McAvoy and Abraham Snyder for support and development of functional data analysis procedures. Enami Yasui provided assistance with data collection. We thank Francis Miezin for helpful discussion.

Correspondence should be addressed to Katerina Velanova, 121 Meyran Avenue, Loeffler Building, Room 109, University of Pittsburgh, Pittsburgh, PA 15213. E-mail:velanovak@upmc.edu.

D01:10.1523/JNEUROSCI.1579-09.2009

Copyright $\odot 2009$ Society for Neuroscience 0270-6474/09/2912558-10\$15.00/0 ilar to Braver et al. (2003) and Dosenbach et al. (2006)] and distinguish such activation from preparatory activation that may or may not be sustained.

Mixed block/event-related or "hybrid design" functional magnetic resonance imaging (fMRI) has proven highly effective for distinguishing simultaneously occurring sustained and transient signals (Visscher et al., 2003). In the present study, we make use of these methods to estimate sustained and transient activation elicited during performance of the antisaccade (AS) task, an oculomotor test of inhibitory control (Hallett, 1978), at three stages of development: late childhood, adolescence, and young adulthood.

Our primary aim was to describe the development of sustained processes. We hypothesized that regions belonging to a "core" task set network, including medial superior frontal/anterior cingulate cortex $(\mathrm{msF} / \mathrm{aCC})$ and anterior insula/frontal operculum (aI/fO) would show similar response profiles across age groups, consistent with their playing a fundamental role in all ongoing task performance (Dosenbach et al., 2006, 2008). However, we anticipated that regions implicated in task-specific inhibitory set maintenance would show continued specialization beyond childhood, and a more protracted developmental trajectory than that of transient controlled processes, analogous to recent resting-state functional connectivity ( $r$-fcMRI) findings (Fair et al., 2007). Our second aim was to assess the contribution of sustained processes to age-related improvements in performance. In particular, we hypothesized that sustained processes would play a central role in the transition from adolescent- to adult-level performance. At this transition, we have shown that transient signaling associated with AS control functions has stabilized, yet performance continues to improve into adulthood (Velanova et al., 2008). Thus, the trajectory of improved performance with age is not fully explained by transient modulations. 
In sum, our goal was to highlight the importance of supervisory control functions and their critical role in the attainment of adult-level inhibitory control.

\section{Materials and Methods}

Correct performance of an AS trial requires inhibition of a prepotent saccade toward a briefly presented peripheral stimulus and, instead, execution of a voluntary saccade toward the empty mirror location. In this study, during fMRI, AS and prosaccade (PS) trials (requiring only a saccade toward a peripheral stimulus) were presented in blocks interspersed with extended periods of fixation. This arrangement allowed the estimation of sustained brain activation maintained throughout epochs of AS performance, as well as transient activation associated with individual (correctly performed) AS trials.

\section{Participants}

Ninety-eight individuals aged 8-27 years participated in accordance with University of Pittsburgh Institutional Review Board guidelines: 35 were children aged $8-12$ years, 35 were adolescents aged $13-17$ years, and 28 were adults aged $18-27$ years. No standards exist for defining child and adolescent age ranges; thus, groups were defined based on our past behavioral studies indicating differential cognitive performance (Luna et al., 2004). Participants were native English speakers with no history of neurological or psychiatric problems in themselves or a first-degree relative. Three were left-handed. Vision was normal or corrected to normal using magnet compatible glasses or contact lenses. Sixteen participants either failed to complete the study or produced data with sufficient artifacts to preclude additional analysis. Four participants were excluded because of equipment-related failure. Thus, we report data from 78 participants (26 children: mean age, 10.50; SD, 1.39; 25 adolescents: mean age, 15.32; SD, 1.63; and 27 adults: mean age, 20.74; SD, 2.75). Seventyseven of these participants contributed (transient) data reported by Velanova et al. (2008). The 78th participant performed at ceiling, and thus their data was not included in our previous report (which was concerned with responses to errors).

Participants were trained on the scanned task in the behavioral laboratory within 3 months of $\mathrm{AMRI}$, at which time they also completed assessments of handedness (Annett, 1967; Oldfield, 1971) and intelligence (Wechsler Abbreviated Scale of Intelligence) (The Psychological Corporation, 1999). Immediately before scanning, naive participants spent $\sim 15 \mathrm{~min}$ in a mock scanner to acclimate them to the magnetic resonance (MR) environment (Rosenberg et al., 1997).

\section{fMRI data acquisition}

Data were acquired using a Siemens 3 tesla MAGNETOM Allegra fitted with a standard circularity-polarized head coil. Pillows and tape minimized head movement. Earplugs dampened scanner noise. A personal computer (Dell Dimension 8200, Pentium 4, 2 GHz, Windows XP) running E-Prime (Psychology Software Tools) controlled stimulus display. Stimuli were projected onto a screen at the head of the scanner bore viewable via a mirror attached to the head coil.

Structural images were acquired first using a sagittal magnetizationprepared rapid gradient echo (MP-RAGE) T1-weighted sequence [repetition time (TR), $1570 \mathrm{~ms}$; echo time (TE), $3.04 \mathrm{~ms}$; flip angle $(a), 8^{\circ}$; inversion time (TI), $800 \mathrm{~ms}$; voxel size, $0.78125 \times 0.78125 \times 1 \mathrm{~mm}$ ]. Functional images were then acquired using an echoplanar sequence sensitive to blood oxygen level-dependent (BOLD) contrast $\left(\mathrm{T}^{*}\right)$ (TR, $1.5 \mathrm{~s}$; TE, $25 \mathrm{~ms} ; a=70^{\circ}$; voxel size, $3.125 \times 3.125 \mathrm{~mm}$ in-plane resolution) (Kwong et al., 1992; Ogawa et al., 1992). Participants performed four functional runs (each, 6 min $15 \mathrm{~s}$ ), followed by up to three runs of an unrelated experiment. During each run, 29 contiguous 4-mm-thick axial images were acquired parallel to the anterior commissure-posterior commissure plane. The first six images in each run were discarded to allow stabilization of longitudinal magnetization.

\section{Behavioral paradigm}

During each run, participants alternated between blocked periods of oculomotor task performance and blocked periods of fixation (Chawla et al., 1999; Donaldson et al., 2001; Velanova et al., 2003). Each run con- sisted of $36 \mathrm{~s}$ (24 MR frames) of fixation (control), followed by a $114 \mathrm{~s}$ task block (76 frames), a second block of fixation (45 s; 30 frames), a second task block (114 s; 76 frames), and a final block of fixation ( $36 \mathrm{~s} ; 24$ frames). Participants performed the AS task during one task block and a PS task during the second. The order of tasks was counterbalanced across runs (within participant) and across participants. Participants were explicitly told the task order before each run commenced.

Each task block was preceded by a $3 \mathrm{~s}$ cue informing participants about the nature of the upcoming trials (either "Start LOOK-AWAY game" for AS blocks, or "Start LOOK-TOWARD game" for PS blocks). Twelve AS trials or 12 PS trials were presented in each task block, such that, across four runs, participants performed 48 AS and 48 PS trials. Intervals between trials were planned so that the time between trials (during which a white fixation crosshair was presented) varied from 3 to $9 \mathrm{~s}$ (two to six frames) and was more often shorter than longer (Dale and Buckner, 1997; Dale, 1999). This temporal jitter allowed separation of sustained and trial-related signal components and differed from trial to trial for each participant. Trial presentation was time-locked to the onset of successive whole-brain image acquisitions. Each task block ended with a $3 \mathrm{~s}$ "task end" cue, alerting participants that a long period of fixation would follow. Three additional frames of fixation served to jitter the onset of the task block proper (after cue presentation) and were arranged such that, if three additional frames $(4.5 \mathrm{~s}$ ) of fixation followed the task onset cue, no additional fixation frames preceded the task end cue, else if two additional frames $(3 \mathrm{~s})$ of fixation followed the task onset cue, one frame (1.5 s) preceded the task end cue, and so forth. Inclusion of these additional frames of fixation reduced noise in estimating responses associated with task start and end cues, and also modestly improved estimates of transient and sustained effects.

AS and PS task trials began with a 3 s colored fixation crosshair (subtending $\sim 0.7^{\circ}$ of visual angle) instructing participants to make a prosaccade (green) or an antisaccade (red). This was followed immediately by a $1.5 \mathrm{~s}$ peripheral saccade stimulus. No "gap" was interposed between the instruction cue and saccade stimulus to increase the probability of accurate performance in younger participants (Fischer and Weber, 1997). Each target stimulus was a yellow spot, subtending $\sim 0.5^{\circ}$, presented on the horizontal meridian at one of six eccentricities (at $\pm 3,6$, or $9^{\circ}$ ). Target location order was randomized within each task block. For PS trials, participants' task was to look toward the saccade stimulus. For AS trials, participants were instructed to inhibit saccades toward the saccade stimulus and to look instead toward the empty location directly opposite. Run and task trial structures are depicted in Figure 1 [Velanova et al. (2008), their Fig. 1, reprinted with permission]. Data from AS trials and AS task blocks only are reported here.

\section{Eye tracking}

Eye movement measurements were obtained during scanning using a long-range optics (LRO) eye-tracking system (model R-LRO6; Applied Science Laboratories). Nine-point calibrations were performed at the beginning of the session and between runs as necessary. Eye movement data were analyzed and scored off-line using ILAB (Gitelman, 2002) in conjunction with an in-house scoring suite. Eye movements occurring after the presentation of target saccade stimuli were scored for performance accuracy (as correct, incorrect, and scoring omissions for each trial type) and latency.

\section{fMRI data preprocessing}

Data were preprocessed to remove noise and artifacts. Motion was corrected within and across runs using a rigid-body rotation and translation algorithm (Snyder, 1996). Image slices were realigned in time to the midpoint of the first slice using sinc interpolation. Data were normalized to a whole-run modal magnitude of 1000 (Ojemann et al., 1997) and were resampled into a standardized atlas space (see below) (Talairach and Tournoux, 1988) using $2 \mathrm{~mm}$ isotropic voxels (see Maccotta et al., 2001, for details of the warping method). Atlas registration involved using a series of affine transforms to align each subject's T1-weighted image to an atlas-transformed target T1-weighted template (Michelon et al., 2003). The template was derived from MP-RAGE images obtained from 12 independently scanned neurologically normal young adults 
(see Kang et al., 2003). Difference image variance minimization was the objective function (Snyder, 1996). This method has been validated for the comparison of children as young as 7 years with adults at BOLD image resolutions $>5 \mathrm{~mm}$ (Burgund et al., 2002; Kang et al., 2003) and differs from statistical parametric mapping (SPM) atlas registration (with affine normalization) only in the image used as the atlas-representative target.

\section{Movement analysis}

Measures of head movement were obtained from the output of the rigid-body rotation and translation algorithm. Translations and rotations in the $x, y$, and $z$ dimensions were averaged across frames, and total root mean square (RMS) linear and angular precision measures were calculated for each run. Runs in which a participant's total RMS movement exceeded 1 $\mathrm{mm}$ or degree were excluded from additional analysis. Four runs of data were included for 26 of the 27 adult participants, 24 of the 25 adolescents, and for 17 of the 26 child participants. The remainder contributed three runs. Values for included runs were averaged for each participant, and ANOVAs were performed, testing for differences between age groups. RMS movement for all groups was considerably below the a priori selected cutoff of $1 \mathrm{~mm}$ or degree. Children, however, showed significantly more head movement on average $(0.39)$ than did adolescents or adults (both 0.26; main effect of age group, $F_{(2,75)}=9.44, p<0.001$ ).

\section{fMRI data analyses}

Preprocessed data were analyzed using the general linear model (GLM) (Friston et al., 1995; Worsley and Friston, 1995; Zarahn et al., 1997). Analyses were performed to estimate sustained signal changes maintained throughout AS (and PS) blocks, and transient signal modulation during AS (and PS) trials using fIDL software (Miezin et al., 2000; Ollinger et al., 2001a,b). Additionally, regressors were coded into the GLM to take into account task start and end cues, within-run drift (linear trend terms), and run means (run-wise constant terms) (Velanova et al., 2003; Visscher et al., 2003). Effects for all analyses are described in terms of percentage signal change, defined as signal magnitude divided by the mean of the estimated constant terms.

Sustained effects. Two sustained effects, one corresponding to each block type (AS and PS), were coded in the GLM. These were coded as single regressors with an assumed response shape (specifically, a square wave function starting $19.5 \mathrm{~s} / 14$ time points after the cues at the beginning of each task block and terminating at the end of each task epoch) (Dosenbach et al., 2006; Fair et al., 2007). A single magnitude estimate (relative to baseline) for each sustained effect was computed for each block condition at each voxel. For this report, magnitude estimates for AS sustained activation (only) were entered into voxel-by-voxel $t$ tests and one-way ANOVA, which compared the magnitude of sustained activation to baseline within age groups (and across all participants), and compared age groups, respectively. Exploratory statistical images resulting from these analyses were produced by converting the observed $t$ and $F$ statistics to $z$ statistics, plotting them across the brain, smoothing with a Gaussian spatial filter of $8 \mathrm{~mm}$ full width at half-maximum, excluding voxels with values of $z<2$ and then voxels falling outside clusters composed of a minimum of 100 voxels. Estimates of AS sustained activation relative to baseline were also entered into region-specific analyses, as below.

Transient effects. For each participant, the BOLD response to each trial type (i.e., correct responses, incorrect responses, and scoring omissions for both AS and PS trials) was estimated by coding a different regressor (delta function) for each of the 15 time points (i.e., image acquisitions) after each trial onset. This produced one time course estimate (over 15 time points) per voxel, for both AS and PS trials. Cross-correlation magnitudes were then computed for correctly performed AS trials (for each participant) using the inner product of the estimated AS time course and a vector of contrast weights modeling the hemodynamic response func-
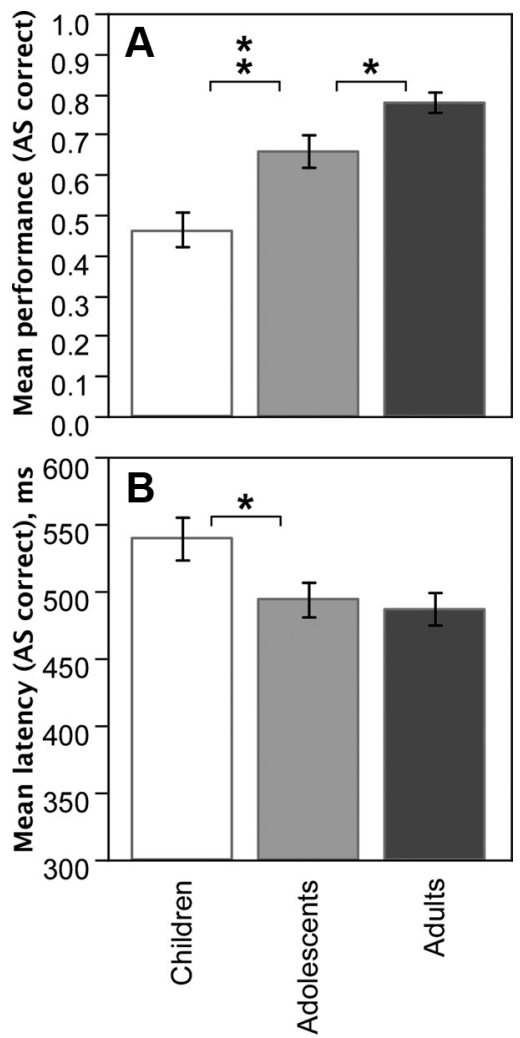

Age group

Figure 2. A, Mean probability of correct AS trial performance as a function of age group. $B$, Mean initial saccade latencies for correctly performed AS trials as a function of age group. Asterisks indicate the significance of (adjacent) group comparisons: ${ }^{*} p<0.05$; ${ }^{* *} p<0.01$. Error bars indicate SEM.

tion (i.e., a gamma function; delay, $2 \mathrm{~s}$; time constant, $1.25 \mathrm{~s}$ ). These summary cross-correlation magnitudes were entered into ANOVA comparing age groups from which exploratory statistical images based on $F$ statistics were derived. Again, these were smoothed, thresholded, and corrected as above $(z=3 ; p<0.05)$ (Forman et al., 1995; McAvoy et al., 2001). We note that, in common with previous reports (Visscher et al., 2003; Burgund et al., 2006; Dosenbach et al., 2006), differing thresholds were applied for sustained and transient effects based on their reflecting different types of neural signals that are known to show substantial differences in statistical reliability and signal magnitude. 

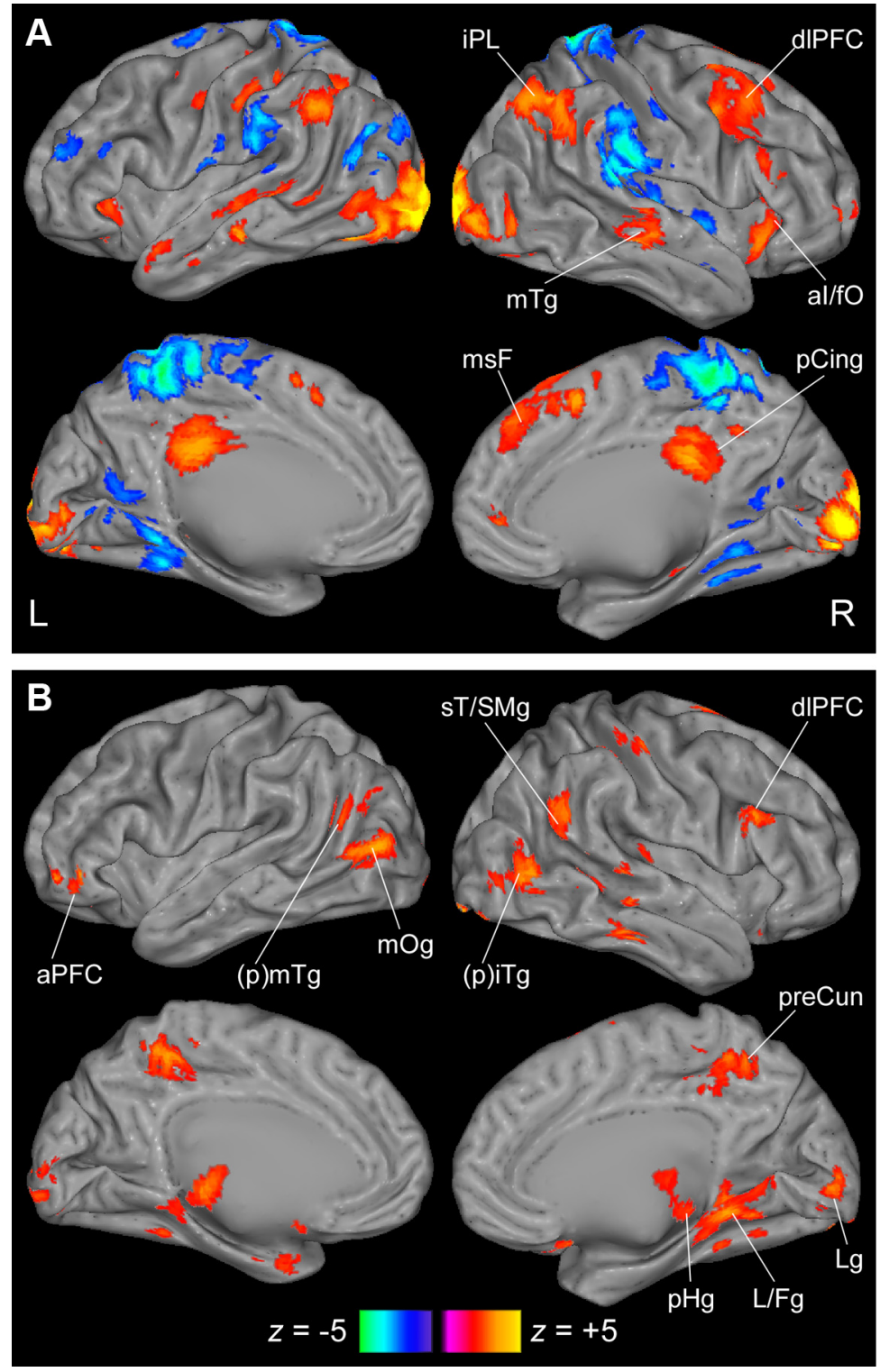

Figure 3. Voxels showing sustained statistical activation change during AS task blocks relative to baseline displayed on partially inflated lateral and medial cortical surfaces. Left and right hemisphere surfaces appear to the left and right. Clusters of activation smaller than 100 contiguous voxels are not displayed. Images are thresholded at $z=2 . A,(t) z$-statistical map of voxels showing sustained activation across all participants independent of age group. $B$, (F)z-statistical map of voxels showing age group differences in sustained activation during AS blocks. Note that, because this map is based on ANOVA, no direction of effects is indicated.

\section{Analyses in regions of interest}

Functional regions of interest (ROIs) showing developmental changes in sustained and transient activation (separately) were identified by taking the uncorrected $(F) z$-statistical image depicting voxels in which there was a main effect of age group for AS sustained activation and AS transient activation, preblurring using a $4 \mathrm{~mm}$ hard-sphere kernel, and applying an automated (local extremum) search algorithm that located peaks exceeding a significance threshold of $z=2$ for sustained effects and $z=3$ for transient effects. Peaks separated by $<10 \mathrm{~mm}$ were consolidated by averaging coordinates. Regions were defined by including all voxels that also appeared in the relevant corrected statistical image and that were within a $10 \mathrm{~mm}$ radius of each identified peak, and then discarding clusters that comprised $<100$ contiguous voxels. This procedure defined ROIs that changed activation based on the age group effect represented in the base exploratory statistical image. For each region thus defined, relevant response magnitudes were averaged across all voxels (within subject), and submitted to analyses paralleling those conducted on a voxel-by-voxel basis, together with correlational analyses (based on Pear- son's $r$ ) assessing relationships between the estimated magnitude of sustained effects and participant's age and level of performances, measured in terms of the rate of correct AS trial performance. Supplemental analyses were also conducted to visualize the temporal evolution of sustained effects and served to confirm their sustained nature. These are presented in supplemental Figure 3 (available at www.jneurosci.org as supplemental material).

\section{Results}

\section{Behavioral results}

Rates of correct AS trial performance, when participants successfully inhibited saccades toward peripherally presented stimuli, increased with age (main effect of age group; $F_{(2,75)}=18.51 ; p<$ $0.0001)$. Consistent with previous findings (Fischer et al., 1997; Munoz et al., 1998; Fukushima et al., 2000; Klein and Foerster, 2001; Luna et al., 2004; Irving et al., 2006), AS trial performance improved significantly from childhood to adolescence $\left(t_{(49)}=3.25 ; p<0.01\right)$ and from adolescence to adulthood $\left(t_{(50)}=2.55\right.$; $p<0.05$ ) (Fig. 2A). As anticipated, rates of correct performance for PS trials were at ceiling (0.99) and did not differ among age groups (main effect of age group; $\left.F_{(2,75)}=1.04 ; p>0.35\right)$. Similarly, rates of corrective saccades toward the target location on AS error trials [i.e., when participants initially failed to inhibit (erroneous) prosaccades] did not differ among age groups (mean correction rate, $0.93 ; F_{(2,74)}$ $=1.33 ; p>0.27)$, suggesting that task instructions were understood and followed by all age groups, despite their showing differing levels of performance. The high rate of corrective saccades is particularly important when considering children's performance as it indicates that even these participants were on-task and had some intention to inhibit. This was confirmed by responses to a follow-up questionnaire.

Latencies for initial saccades on correctly performed AS trials were significantly longer for children than for adolescents and adults (main effect of age group; $F_{(2,75)}=4.63 ; p<0.02$ ), who did not differ (Luna et al., 2008) (Fig. $2 B$ ). Mean AS latencies for all groups were within one repetition time (TR/whole-brain image acquisition). Full-scale intelligence quotient (IQ) differed marginally between groups (children, 107.5; adolescents, 108.3, adults, $113.4 ; F_{(2,70)}=2.65 ; p>$ $0.07)$, with the marginal effect driven by the difference between children and adults.

\section{fMRI results}

\section{Regions showing sustained activation}

As a starting point, we sought to distinguish regions showing similar levels of sustained activation across development from those showing differing levels of activation. Figure 3 shows the results of whole-brain exploratory analyses conducted with this aim in mind. Consistent with previous reports, across partici- 
pants and independent of age group (see Fig. 3A), positive sustained activation relative to baseline was observed in a network of regions prominently including $\mathrm{msF}$, bilateral $\mathrm{aI} / \mathrm{fO}$, inferior parietal lobule (iPL), and middle temporal gyrus (mTg) —regions specifically identified as routinely showing sustained activation across cognitive tasks by Dosenbach et al. (2006) (for comparison, see their Fig. 4) in their meta-analytic review of 10 studies conducted within their laboratory. Also in keeping with previous findings, negative sustained activation was observed in left posterior temporal/occipital and middle occipital cortex, and bilateral anterior fusiform gyrus. (For interested readers, we present a parallel map showing sustained activation during PS task blocks in supplemental Fig. 1, available at www.jneurosci. org as supplemental material.)

Notable differences from previous reports included; positive activation in right dorsolateral prefrontal cortex (dlPFC), bilateral dorsal posterior cingulate (pCing), and striate cortex, and negative activation along bilateral postcentral gyrus and in paracentral lobule. Also, conspicuously absent given past reports was negative sustained activation in ventromedial prefrontal cortex (vmPFC). Rather, as reported by Velanova et al. (2008) and Polli et al. (2005), negative activation in vmPFC was a trial-specific transient response. These differences from previous reports, we suggest, are attributable to our uniquely having measured sustained activation during performance of a task that depends strongly on participants exerting oculomotor control.

To assess specific patterns of sustained activation, regions of interest composed of a minimum of 100 contiguous $2 \mathrm{~mm}$ isotropic voxels were derived from the map shown in Figure $3 \mathrm{~A}$, and estimates of the magnitude of sustained activation maintained throughout AS blocks for children, adolescents, and adults were computed and compared. These common regions showed similar levels of sustained activation across age groups except in left lingual gyrus [near Brodmann area (BA) 17] $\left(F_{(2,75)}=\right.$ $4.12 ; p<0.05)$. This region was represented in the $(F) z$ statistical map showing voxels in which a significant main effect of age group was observed (Fig. 3B) and thus is discussed (below) in that context. The similarity of activation in the remainder of regions, however, suggests, in consensus with other reports (Fair et al., 2007), that a critical set of regions supports sustained processing independent of age and level of performance.

\section{Developmental effects}

The $(F) z$-statistical map showing voxelwise age group differences in sustained activation is presented in Figure $3 B$. Regions of interest composed of $\geq 100$ voxels were also derived from this map (Fig. 4A), and again, magnitudes of sustained activation were estimated within each region for each age group. Regions were then subdivided into groups based on two criteria: (1) the principal direction of activation (whether positive or negative relative to baseline) across age groups and (2) the relationship between endpoint (i.e., child and adult) age groups (whether adults $>$ children or vice versa). All regions could be categorized as falling into three groups: (1) "white" regions showing sustained negative activation that was attenuated (i.e., became less negative) with age, (2) "black" regions showing (initial) sustained positive activation (in childhood) that decreased with age, and (3) "red" regions showing sustained positive activation that increased with age. Regions falling into each group are listed and described in Table 1 and shown in Figure $4 A$. ANOVA comparing age groups across regions within each region group confirmed the similarity of the developmental pattern of activation in regions comprising each region group (region $\times$ age group interaction for

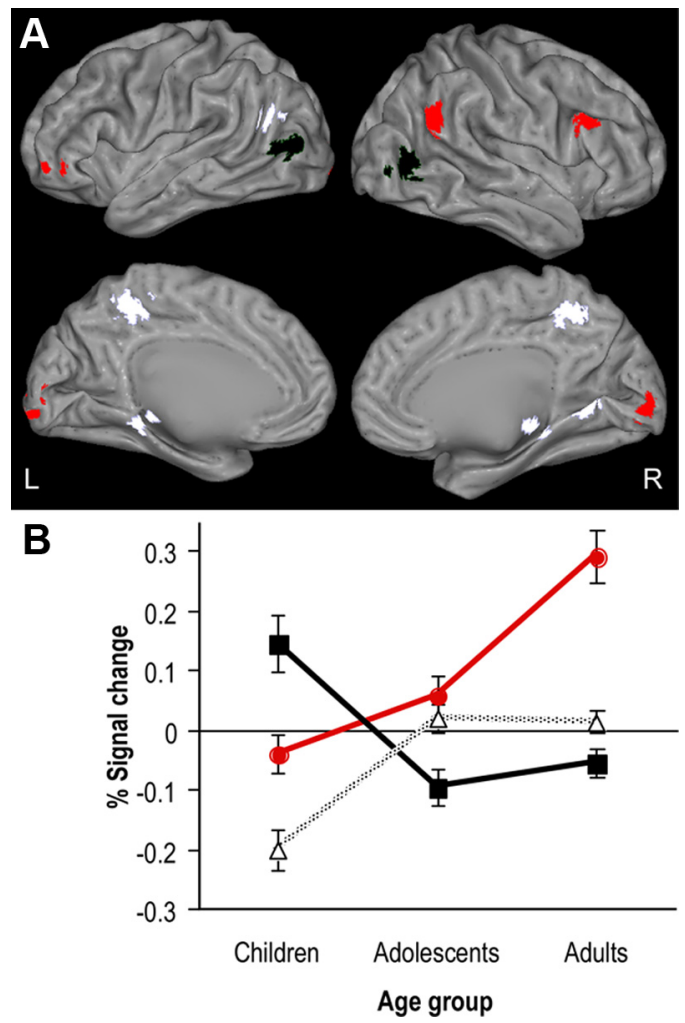

Figure 4. Regions derived from the $F(z)$-statistical map of voxels showing differential magnitudes of sustained activation between age groups. $\boldsymbol{A}$, Regions displayed on partially inflated cortical surfaces (similar to Fig. 2). Regions are classified as falling into one of three groups and are color-coded in white, black, or red to represent that classification. Region characteristics are provided in Table 1. B, Mean percentage sustained signal change for white, black, and red regions as a function of age group. Error bars indicate SEM.

white regions showing attenuated negative activation with age, $F_{(12,450)}=1.23, p=0.26$; for black regions showing attenuated positive activation with age, $F_{(2,75)}=0.74, p=0.48$; and for red regions showing increased positive activation with age, $F_{(8,300)}=$ $1.64, p=0.11)$. ANOVA comparing age groups across region groups indicated that groups of regions (as defined) were statistically dissociable (region group $\times$ age group interaction, $\left.F_{(4,150)}=23.20, p<0.000001\right)$.

Of note, two groups of regions (white and black regions) showed a reduced absolute magnitude of sustained activation in adults (and adolescents) relative to children (Fig. 4B). Indeed, when the magnitude of sustained activation shown by adults within regions comprising these two groups was compared with baseline, only right parahippocampal gyrus showed significant activation in adults that differed from baseline $\left(t_{(26)}=2.24 ; p<\right.$ $0.05)$. The remainder of white and black regions made only marginal (black regions; both $t_{(26)}=1.97 ; p=0.06$ ) or nonsignificant contributions to mature performance. This was in sharp contrast to red regions, which showed increasing positive sustained activation with age, all of which were sites of significant sustained activation in adults $\left(t_{(26)}=2.96-4.15 ; p=0.007-0.0003\right)$ (supplemental Figs. 2C, 3, available at www.jneurosci.org as supplemental material) that consistently differed from adolescents $\left(t_{(50)}=2.16-3.56 ; p=0.04-0.0008\right)$ as well as children $\left(t_{(51)}=3.39-4.09 ; p=0.001-0.0002\right)$. These results suggest that, in regions contributing to mature performance, which also show developmental change [i.e., right dlPFC, left anterior prefrontal cortex (aPFC), right superior temporal/parietal cortex (sT/sMg), and bilateral occipital cortex along 
Table 1. Characteristics of regions comprising white, black, and red region groups displaying developmental changes in sustained (AS task block-related) signal

\begin{tabular}{|c|c|c|c|c|c|c|c|}
\hline Region group & Region & Approximate BA & $x$ & $y$ & $Z$ & $n$ voxels & z at peak \\
\hline \multirow[t]{7}{*}{ White } & L parietal: precuneus & 7 & -7 & -44 & 48 & 323 & 3.24 \\
\hline & R parietal: precuneus (preCun) & 7 & 8 & -52 & 47 & 236 & 2.83 \\
\hline & L temporal/occipital: (posterior) middle temporal gyrus $[(\mathrm{p}) \mathrm{mTg}]$ & 39 & -42 & -63 & 17 & 155 & 2.58 \\
\hline & R medial occipital: lingual/fusiform gyrus (L/Fg) & 19 & 10 & -57 & 1 & 148 & 2.36 \\
\hline & L parahippocampal gyrus & 30 & -15 & -37 & -6 & 113 & 2.10 \\
\hline & R parahippocampal gyrus (pHg) & $27 / 30$ & 19 & -31 & -4 & 133 & 2.36 \\
\hline & R thalamus: pulvinar (not depicted in Fig. 3) & & 3 & -27 & 3 & 133 & 2.46 \\
\hline \multirow[t]{2}{*}{ Black } & L occipital: middle occipital gyrus (m0g) & 19 & -48 & -76 & 5 & 338 & 3.40 \\
\hline & R occipital: inferior temporal gyrus [(p)iTg] & 19 & 50 & -68 & 1 & 281 & 3.13 \\
\hline \multirow[t]{5}{*}{ Red } & L frontal: middle frontal gyrus (aPFC) & $10 / 47$ & -46 & 40 & 3 & 118 & 2.93 \\
\hline & R frontal: middle frontal gyrus (dIPFC) & $9 / 44$ & 51 & 16 & 29 & 162 & 2.92 \\
\hline & R temporal/parietal: superior temporal/supramarginal gyrus (sT/SMg) & $39 / 40$ & 53 & -55 & 24 & 286 & 2.93 \\
\hline & L occipital: lingual gyrus & $17 / 18$ & -11 & -96 & -6 & 219 & 2.69 \\
\hline & R occipital: lingual gyrus (Lg) & 17 & 11 & -92 & -1 & 123 & 2.71 \\
\hline
\end{tabular}

L, Left; $R$, right.

Table 2. Correlations between the magnitude of sustained activation, age, and performance observed within individual red regions

\begin{tabular}{lll}
\hline Region & $r_{\text {sustained activation, age }}$ & $r_{\text {sustained activation, performance }}$ \\
\hline L frontal: middle frontal gyrus (aPFC) & $0.26^{*}$ & $0.19^{\mathrm{m}}$ \\
R frontal: middle frontal gyrus (dIPFC) & $0.34^{* *}$ & $0.34^{* *}$ \\
R temporal/parietal: superior temporal/supramarginal gyrus (sT/SMg) & $0.34^{* *}$ & $0.31^{* *}$ \\
L occipital: lingual gyrus & $0.37^{* * *}$ & $0.29^{*}$ \\
R occipital: lingual gyrus (Lg) & $0.31^{* *}$ & $0.42^{* * *}$ \\
\hline
\end{tabular}

L, Left; $R$, right.

$\mathrm{m}_{p}<0.10,{ }^{*} p<0.05,{ }^{* *} p<0.01,{ }^{* * *} p<0.001$.

lingual gyrus], maturation is protracted, extending beyond childhood from adolescence to adulthood.

Correlational analyses: sustained effects

The observation that the maturation of sustained activation is protracted is further supported by correlational analyses showing robust associations between age (treated as a continuous variable) and the magnitude of sustained activation in (red) regions that contribute to mature performance. When these regions were considered as a single cluster, not only was the association between age and sustained activation robust $\left(r_{(76)}=0.52 ; p<\right.$ 0.00001 ), but it remained so when performance (measured in terms of the proportion of correctly performed AS trials) was partialled out $\left(r_{(75)}=0.36 ; p<0.01\right)$.

To establish that these effects were not driven by endpoint groups, similar analyses were conducted with the range of ages restricted to those spanning the adolescent and adult age groups. Again, a significant positive relationship between age and sustained activation was observed $\left(r_{(50)}=0.36 ; p<0.01\right)$ and remained significant when performance served as a covariate $\left(r_{(50)}=0.30 ; p<\right.$ $0.05)$. In every case, linear regression curves provided better fits than quadratic, cubic, logarithmic, or exponential functions.

Although we give preference to analyses conducted across the region group because of the increased stability of estimates of sustained signal magnitude, we also note that each individual region comprising the (red) region group showed a robust correlation between age and magnitude of sustained activation $\left(r_{(76)}=0.26-0.37 ; p<0.02-0.0009\right)$. Significant relationships between performance and sustained activation were also observed $\left(r_{(76)}=0.29-0.42 ; p<0.01-0.0002\right)$, except in left aPFC, in which this relationship was only marginally significant $\left(r_{(76)}=\right.$ $0.19 ; p=0.08)$. Individual regions, excluding right lingual gyrus, also showed significant or marginally significant associations between age and sustained activation when performance was partialled out $\left(r_{(75)}=0.19-0.27 ; p<0.10-0.01\right)$ (for correlations observed within specific regions, see Table 2$)$.

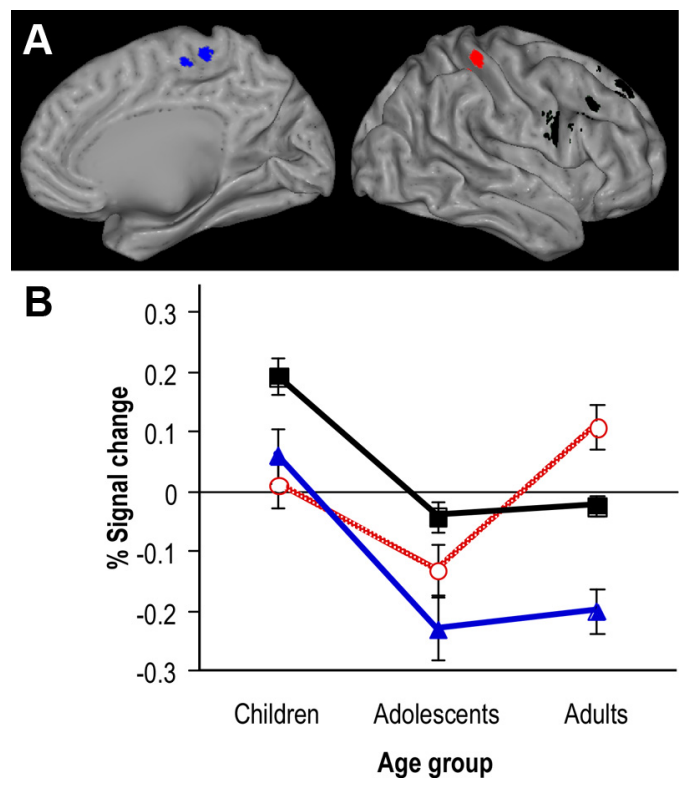

Figure 5. Regions derived from the $F(z)$-statistical map of voxels showing differential magnitudes of transient (correct AS trial-specific) activation between age groups. $\boldsymbol{A}$, Regions displayed on partially inflated right hemisphere (medial and lateral) cortical surfaces. Regions are classified as falling into one of three groups and are color-coded in blue, black, or red' to represent that classification. Region characteristics are provided in Table 3. $\boldsymbol{B}$, Mean percentage sustained signal change for "blue ${ }^{\top}$, " "black ${ }^{\top}$," and "red' ${ }^{\top}$ " regions as a function of age group. Error bars indicate SEM.

\section{Comparisons with transient activation associated with correctly} performed AS trials

The developmental trajectory of sustained activation differed from that for transient activation. Paralleling the approach taken to ascertain developmental changes in sustained activation, regions of interest for transient effects were derived from the $(F) z$-statistical map showing voxelwise age group differences in 


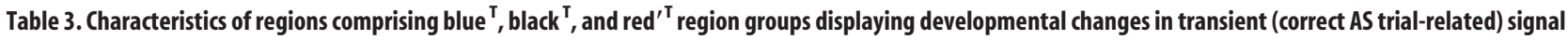

\begin{tabular}{llcrrrrr}
\hline Region group $^{\prime}$ & Region & Approximate BA & $x$ & $y$ & $z$ & $n$ voxels & $z$ at peak \\
\hline Black $^{\top}$ & R frontal: superior frontal gyrus & 8 & 26 & 32 & 47 & 199 & 3.26 \\
& R frontal: middle frontal gyrus & 9 & 38 & 14 & 31 & 106 & 3.10 \\
& R frontal: inferior frontal gyrus & $9 / 44$ & 55 & 4 & 25 & 202 & 3.21 \\
Blue $^{\top}$ & L/R frontal: paracentral lobule & 5 & 0 & -30 & 53 & 267 & 3.95 \\
Red $^{\prime \top}$ & R parietal: inferior parietal lobule/postcentral gyrus (iPL/pCG) & 40 & 42 & -36 & 50 & 124 & 3.15 \\
\hline
\end{tabular}

L, Left; R, right.

transient activation elicited during correctly performed AS trials relative to baseline. Five regions were identified as showing developmental change: three in frontal cortex along right superior, middle, and inferior frontal gyri, together with regions in paracentral lobule and right inferior parietal lobule/postcentral gyrus (iPL/pcG) (Fig. 5A, Table 3). Regions were again classified according to direction of activation and relationships between endpoint age groups. All frontal regions showed positive trial-related activation that decreased from childhood to adulthood (Fig. 5, black regions). In these regions, activation in adolescents and adults (who did not differ; $t_{(50)}=-0.39-0.98 ; p=0.70-0.33$ ) showed significant divergence from baseline only in superior frontal gyrus $\left(t_{(24)}=-2.31, p=0.03\right.$ for adolescents; and $t_{(26)}=$ $-2.23, p=0.04$ for adults). In paracentral lobule (Fig. 5, blue), both adolescents and adults showed significantly decreased transient activation relative to children $\left(t_{(49)}=-4.18, p<0.0001\right.$ for adolescents vs children; and $t_{(51)}=-4.44, p<0.0001$ for adults vs children), who did not differ from baseline $\left(t_{(25)}=1.30 ; p=\right.$ $0.21)$. Again, there was no significant difference in activation between adolescents and adults $\left(t_{(50)}=0.49 ; p=0.63\right)$. Finally, in iPL/pcG (Fig. 5, red'), the trajectory of developmental effects was nonlinear; activation in children did not differ from baseline $\left(t_{(25)}=0.29 ; p=0.77\right)$, activation in adolescents was decreased $\left(t_{(24)}=-3.16 ; p=0.004\right)$, and transient activation in adults was modestly although significantly increased relative to baseline $\left(t_{(26)}=2.90 ; p=0.007\right)$. Although all age groups differed, the difference between adults and children was only marginal $\left(t_{(51)}=\right.$ $1.72 ; p=0.09)$. The larger message, however, is that beyond $\mathrm{iPL} / \mathrm{pcG}$, no differences were observed between adolescents and adults, suggesting that transient activation is mostly mature by adolescence-despite continuing improvement in performanceand in contrast to their being continued increases in sustained activation in a critical subset of regions.

\section{Discussion}

The aim of the present study was to illustrate the critical contribution of sustained task set-related activation to the maturation of inhibitory control. Consistent with previous findings, our results indicate that typically developing individuals are able to successfully produce at least some inhibitory responses by 8 years of age. Importantly, what improves with age, even through young adulthood, is the rate of successful inhibitory responding (Fischer et al., 1997; Munoz et al., 1998; Klein and Foerster, 2001; Luna et al., 2004), suggesting that, for the current task paradigm, processes implicated in sustained performance continue to mature after those supporting trial-specific performance (competence) are in place. Here, we demonstrate that, although transient trialspecific activation is mostly mature by adolescence, sustained brain activation, as evident in adults, follows a more protracted developmental trajectory that mirrors improvements in performance between adolescence and young adulthood. Furthermore, although transient activation in prefrontal regions supporting controlled processing decreases with age, sustained activation in- creases, suggesting a transition in the nature of control functions supporting task performance and, in keeping with previous reports, implicating distinguishable (although related) neural systems having differential developmental profiles.

\section{Developmental change in trial-specific activation provides an insufficient account of age-related improvements in performance between adolescence and adulthood}

Functional imaging studies examining activation associated with effective trial-specific inhibitory control in young adults have consistently demonstrated recruitment of a network of regions prominently including middle and/or inferior prefrontal cortex (Zheng et al., 2008), pre-supplementary motor area (Xue et al., 2008), basal ganglia and thalamic structures (Aron and Poldrack, 2006). Event-related fMRI studies report that AS performance elicits activation in these brain regions (Connolly et al., 2000; Polli et al., 2005; Brown et al., 2006; Curtis and Connolly, 2008), and, in the present study, recruitment of analogous regions was readily evident across age groups (Velanova et al., 2008). Developmental fMRI studies of AS performance, in keeping with studies of inhibitory control using other task paradigms, indicate that age-related changes in activation occur most prominently in prefrontal cortex, particularly on the right (Casey et al., 2000). Results presented here reiterate these findings, with regions in right superior, middle, and inferior frontal cortex showing differential modulation across age groups. Critically, in these regions, the magnitude of activation observed in children was significantly greater than in adolescents or adults. These results are broadly in accord with the hypothesis that children recruit regions within prefrontal cortex to a greater extent to assist with controlled performance (Casey et al., 1997a,b; Durston et al., 2002, 2006; Booth et al., 2003), similar to adults when task performance is experienced as difficult (Mitchell, 2005; Tregellas et al., 2006) or novel (Maccotta and Buckner, 2004; Chein and Schneider, 2005). What is notable with respect to the present study is that the magnitude of transient activation in prefrontal cortex did not differ between adults and adolescents for correctly performed AS trials- despite better overall performance in adults (see Galvan et al., 2006, for analogous findings in orbitofrontal cortex). These results suggest that the processes that support correct (transient) inhibitory responding continue to mature through childhood but are in place by adolescence.

In addition to finding age-dependent activation in prefrontal cortex, regions near the paracentral lobule and in $\mathrm{PL} / \mathrm{pcG}$ showed age-related modulation. Specifically, in paracentral lobule, adolescents and adults showed transient deactivation, whereas children did not-again indicating mature transient signaling by adolescence despite continuing improvement in performance. $\mathrm{iPL} / \mathrm{pcG}$ was unique in that it was recruited differently by the two older age groups; although children showed no significant transient modulation, activation decreased in adolescents but increased in adults. This may suggest that, although adolescents recruit a network of regions similar to adults, modest compensa- 
tory processes may still need to be recruited in adolescence to enable performance of an individual trial in an adult-like manner. More generally, however, beyond $\mathrm{iPL} / \mathrm{pcG}$, patterns of activation in regions showing developmental change indicate that transient activation is mostly mature by adolescence. This finding prompts the question, what mechanisms support improved rates of AS performance between adolescence and adulthood? We contend that sustained processing associated with task set maintenance plays a central role.

We also note that our transient findings occur in the context of correct inhibitory trials. Certainly, children and adolescents generated more errors than adults, suggesting that, although many similarities can be observed in transient activation, task set-related activation may play a role in providing ready access to regions implicated in controlled trial-specific processing.

\section{Sustained activation follows a more protracted developmental trajectory that mirrors performance enhancement from early adolescence through adulthood}

Previous studies have indicated that a specific network of regions supports sustained task set-related processing, prominently including aI/fO, msF/aCC, cuneus/posterior cingulate, and regions within parietal cortex. In particular, Dosenbach et al. (2006, 2008) highlight the central role played by aI/fO and msF/aCC, postulating that these constitute core regions in a network controlling goal-directed behavior through the stable maintenance of task sets. The present data provide a compelling replication of previous findings: across participants, sustained activation was routinely observed in regions similar to those identified by Dosenbach et al. (2006) and others (Velanova et al., 2003).

We also report a small subset of results that differ from those reported by Dosenbach et al. (2006). Of particular note, positive sustained activation was observed in right dlPFC, precuneus/dorsal posterior cingulate, and in striate cortex, and negative sustained activation was absent in vmPFC. We suggest that patterns of activation observed in these regions reflect the strong inhibitory and eye movement control demands of the task under investigation. To our knowledge, no other studies have examined sustained BOLD activation in the context of oculomotor control, and only one other has examined sustained activation associated with inhibitory control (Fassbender et al., 2004). Rather, tasks such as recognition (Velanova et al., 2003; Woodruff et al., 2006), word reading, verb generation, semantic judgment (Dosenbach et al., 2006), and retrieval from working memory (Marklund et al., 2007) form the bulk of tasks previously assessed for sustained activation. Although we hesitate to comment extensively on differences between our own task and others, given the paucity of readily comparable data, we do note that, although decreased sustained activation was absent in vmPFC (even in adults) in the present study (Fig. 3; supplemental Fig. 2, available at www. jneurosci.org as supplemental material), decreased transient activation was observed during correctly performed AS trials (Velanova et al., 2008), consistent with previous reports (Polli et al., 2005). We also acknowledge the suggestion by Dosenbach et al. (2007) that dlPFC may serve as a communication bridge between networks that operate at differing levels of task control, particularly when tasks are cognitively taxing and involve high degrees of conflict as suggested by studies showing strong associations between msF/aCC and dlPFC (Kerns et al., 2004; Kondo et al., 2004).

The principal contribution of this research, however, is our demonstration that, although core regions showing task setrelated signaling did so in a similar manner across age groups, regions implicated in the maintenance of an inhibitory task set showed continued maturation beyond childhood and a more protracted developmental trajectory than that of regions implicated in transient controlled processing. As shown in Figure 4, this maturational process appears to follow two fundamental paths: the first involves the dropping out of regions in precuneus, fusiform, and parahippocampal gyri, and lateral occipitotemporal areas by adolescence. The second path involves a linear increase in activation in frontal and parietal areas, including right dlPFC, left aPFC, and right superior temporal/supramarginal gyrus from childhood to adulthood. Combined, the two paths appear to represent a decrease in reliance on sustained modulation associated with bottom-up occipitotemporal systems and an increase in reliance on sustained processing in top-down frontal and parietal systems.

There was one notable exception to this observation: two regions near the lingual gyrus (near BA 17), which presumably are involved primarily in bottom-up processing, responded similarly to the frontoparietal regions in which activation was positively correlated with age and performance. This somewhat surprising finding may reflect a refinement in the ability to focus on, or bias attentional resources toward (Desimone and Duncan, 1995), processing of the foveated endogenous cue for protracted periods of time.

Additionally, our data support and extend recent rs-fcMRI findings that suggest that sustained and transient task control are modulated by parallel networks (Dosenbach et al., 2007; Fair et al., 2007). Our data suggest that these networks are further distinguishable in that they show differing functional developmental trajectories, of which the sustained task control network provides a basis for continued performance improvement from adolescence to adulthood.

\section{Conclusion}

Our results indicate that there are still important immaturities in adolescence that are specific to the ability to engage regions needed for the efficient and successful maintenance of an inhibitory set. The fact that adolescents still show marked immaturities indicates that important transitions in control functions are still taking place and may provide insight into known vulnerabilities at this stage of development reflected in the emergence of psychopathology and in risk-taking behavior.

\section{References}

Annett M (1967) The binomial distribution of right, mixed and left handedness. Q J Exp Psychol 19:327-333.

Aron AR, Poldrack RA (2006) Cortical and subcortical contributions to Stop signal response inhibition: role of the subthalamic nucleus. J Neurosci 26:2424-2433.

Bedard AC, Nichols S, Barbosa JA, Schachar R, Logan GD, Tannock R (2002) The development of selective inhibitory control across the life span. Dev Neuropsychol 21:93-111.

Booth JR, Burman DD, Meyer JR, Lei Z, Trommer BL, Davenport ND, Li W, Parrish TB, Gitelman DR, Mesulam MM (2003) Neural development of selective attention and response inhibition. Neuroimage 20:737-751.

Braver TS, Reynolds JR, Donaldson DI (2003) Neural mechanisms of transient and sustained cognitive control during task switching. Neuron 39:713-726.

Brown MR, Goltz HC, Vilis T, Ford KA, Everling S (2006) Inhibition and generation of saccades: rapid event-related fMRI of prosaccades, antisaccades, and nogo trials. Neuroimage 33:644-659.

Burgund ED, Kang HC, Kelly JE, Buckner RL, Snyder AZ, Petersen SE, Schlaggar BL (2002) The feasibility of a common stereotactic space for children and adults in fMRI studies of development. Neuroimage 17:184-200.

Burgund ED, Lugar HM, Miezin FM, Schlaggar BL, Petersen SE (2006) The 
development of sustained and transient neural activity. Neuroimage 29:812-821.

Casey BJ, Trainor R, Giedd J, Vauss Y, Vaituzis CK, Hamburger S, Kozuch P, Rapoport JL (1997a) The role of the anterior cingulate in automatic and controlled processes: a developmental neuroanatomical study. Dev Psychobiol 30:61-69.

Casey BJ, Trainor RJ, Orendi JL, Schubert AB, Nystrom LE, Giedd JN, Castellanos FX, Haxby JV, Noll DC, Cohen JD, Forman SD, Dahl RE, Rapoport JL (1997b) A developmental functional MRI study of prefrontal activation during performance of a Go-No-Go task. J Cogn Neurosci 9:835-847.

Casey BJ, Giedd JN, Thomas KM (2000) Structural and functional brain development and its relation to cognitive development. Biol Psychol 54:241-257.

Chawla D, Rees G, Friston KJ (1999) The physiological basis of attentional modulation in extrastriate visual areas. Nat Neurosci 2:671-676.

Chein JM, Schneider W (2005) Neuroimaging studies of practice-related change: fMRI and meta-analytic evidence of a domain-general control network for learning. Brain Res Cogn Brain Res 25:607-623.

Connolly JD, Goodale MA, Desouza JF, Menon RS, Vilis T (2000) A comparison of frontoparietal fMRI activation during anti-saccades and antipointing. J Neurophysiol 84:1645-1655.

Corbetta M, Shulman GL (2002) Control of goal-directed and stimulusdriven attention in the brain. Nat Rev Neurosci 3:201-215.

Curtis CE, Connolly JD (2008) Saccade preparation signals in the human frontal and parietal cortices. J Neurophysiol 99:133-145.

Dale AM (1999) Optimal experimental design for event-related fMRI. Hum Brain Mapp 8:109-114.

Dale AM, Buckner RL (1997) Selective averaging of rapidly presented individual trials using fMRI. Hum Brain Mapp 5:329-340.

Desimone R, Duncan J (1995) Neural mechanisms of selective visual attention. Annu Rev Neurosci 18:193-222.

Diamond A, Goldman-Rakic PS (1989) Comparison of human infants and rhesus monkeys on Piaget's AB task: evidence for dependence on dorsolateral prefrontal cortex. Exp Brain Res 74:24-40.

Donaldson DI, Petersen SE, Ollinger JM, Buckner RL (2001) Dissociating state and item components of recognition memory using fMRI. Neuroimage 13:129-142.

Dosenbach NU, Visscher KM, Palmer ED, Miezin FM, Wenger KK, Kang HC, Burgund ED, Grimes AL, Schlaggar BL, Petersen SE (2006) A core system for the implementation of task sets. Neuron 50:799-812.

Dosenbach NU, Fair DA, Miezin FM, Cohen AL, Wenger KK, Dosenbach RA, Fox MD, Snyder AZ, Vincent JL, Raichle ME, Schlaggar BL, Petersen SE (2007) Distinct brain networks for adaptive and stable task control in humans. Proc Natl Acad Sci U S A 104:11073-11078.

Dosenbach NU, Fair DA, Cohen AL, Schlaggar BL, Petersen SE (2008) A dual-networks architecture of top-down control. Trends Cogn Sci 12:99-105.

Durston S, Thomas KM, Yang Y, Ulug AM, Zimmerman RD, Casey BJ (2002) A neural basis for the development of inhibitory control. Dev Sci 5:F9-F16.

Durston S, Davidson MC, Tottenham N, Galvan A, Spicer J, Fossella JA, Casey BJ (2006) A shift from diffuse to focal cortical activity with development. Dev Sci 9:1-8.

Fair DA, Dosenbach NU, Church JA, Cohen AL, Brahmbhatt S, Miezin FM, Barch DM, Raichle ME, Petersen SE, Schlaggar BL (2007) Development of distinct control networks through segregation and integration. Proc Natl Acad Sci U S A 104:13507-13512.

Fassbender C, Murphy K, Foxe JJ, Wylie GR, Javitt DC, Robertson IH, Garavan H (2004) A topography of executive functions and their interactions revealed by functional magnetic resonance imaging. Brain Res Cogn Brain Res 20:132-143.

Fischer B, Weber H (1997) Effects of stimulus conditions on the performance of antisaccades in man. Exp Brain Res 116:191-200.

Fischer B, Biscaldi M, Gezeck S (1997) On the development of voluntary and reflexive components in human saccade generation. Brain Res 754:285-297.

Forman SD, Cohen JD, Fitzgerald M, Eddy WF, Mintun MA, Noll DC (1995) Improved assessment of significant activation in functional magnetic resonance imaging (fMRI): use of a cluster-size threshold. Magn Reson Med 33:636-647.

Friston KJ, Holmes AP, Poline JB, Grasby PJ, Williams SC, Frackowiak RS,
Turner R (1995) Analysis of fMRI time-series revisited. Neuroimage 2:45-53.

Fukushima J, Hatta T, Fukushima K (2000) Development of voluntary control of saccadic eye movements. I. Age-related changes in normal children. Brain Dev 22:173-180.

Galvan A, Hare TA, Parra CE, Penn J, Voss H, Glover G, Casey BJ (2006) Earlier development of the accumbens relative to orbitofrontal cortex might underlie risk-taking behavior in adolescents. J Neurosci 26:68856892.

Gitelman DR (2002) ILAB: a program for postexperimental eye movement analysis. Behav Res Methods Instrum Comput 34:605-612.

Hallett PE (1978) Primary and secondary saccades to goals defined by instructions. Vision Res 18:1279-1296.

Irving EL, Steinbach MJ, Lillakas L, Babu RJ, Hutchings N (2006) Horizontal saccade dynamics across the human life span. Invest Ophthalmol Vis Sci 47:2478-2484.

Johnson MH (1995) The inhibition of automatic saccades in early infancy. Dev Psychobiol 28:281-291.

Kang HC, Burgund ED, Lugar HM, Petersen SE, Schlaggar BL (2003) Comparison of functional activation foci in children and adults using a common stereotactic space. Neuroimage 19:16-28.

Kerns JG, Cohen JD, MacDonald AW 3rd, Cho RY, Stenger VA, Carter CS (2004) Anterior cingulate conflict monitoring and adjustments in control. Science 303:1023-1026.

Klein C, Foerster F (2001) Development of prosaccade and antisaccade task performance in participants aged 6 to 26 years. Psychophysiology 38:179-189.

Kondo H, Osaka N, Osaka M (2004) Cooperation of the anterior cingulate cortex and dorsolateral prefrontal cortex for attention shifting. Neuroimage 23:670-679.

Kwong KK, Belliveau JW, Chesler DA, Goldberg IE, Weisskoff RM, Poncelet BP, Kennedy DN, Hoppel BE, Cohen MS, Turner R, Cheng H-M, Brady TJ, Rosen BR (1992) Dynamic magnetic resonance imaging of human brain activity during primary sensory stimulation. Proc Natl Acad Sci U S A 89:5675-5679.

Luna B, Garver KE, Urban TA, Lazar NA, Sweeney JA (2004) Maturation of cognitive processes from late childhood to adulthood. Child Dev 75:1357-1372.

Luna B, Velanova K, Geier CF (2008) Development of eye-movement control. Brain Cogn 68:293-308.

Maccotta L, Buckner RL (2004) Evidence for neural effects of repetition that directly correlate with behavioral priming. J Cogn Neurosci $16: 1625-1632$.

Maccotta L, Zacks JM, Buckner RL (2001) Rapid self-paced event-related functional MRI: feasibility and implications of stimulus- versus responselocked timing. Neuroimage 14:1105-1121.

Marklund P, Fransson P, Cabeza R, Petersson KM, Ingvar M, Nyberg L (2007) Sustained and transient neural modulations in prefrontal cortex related to declarative long-term memory, working memory, and attention. Cortex 43:22-37.

McAvoy MP, Ollinger JM, Buckner RL (2001) Cluster size thresholds for assessment of significant activation in fMRI. Neuroimage 13:S198.

Michelon P, Snyder AZ, Buckner RL, McAvoy M, Zacks JM (2003) Neural correlates of incongruous visual information. An event-related fMRI study. Neuroimage 19:1612-1626.

Miezin FM, Maccotta L, Ollinger JM, Petersen SE, Buckner RL (2000) Characterizing the hemodynamic response: effects of presentation rate, sampling procedure, and the possibility of ordering brain activity based on relative timing. Neuroimage 11:735-759.

Mitchell RL (2005) The BOLD response during Stroop task-like inhibition paradigms: effects of task difficulty and task-relevant modality. Brain Cogn 59:23-37.

Monsell S (1996) Control of mental processes. In: Unsolved mysteries of the mind: tutorial essays in cognition (Bruce V, ed), pp 93-148. Hove, UK: Taylor \& Francis.

Munoz DP, Broughton JR, Goldring JE, Armstrong IT (1998) Age-related performance of human subjects on saccadic eye movement tasks. Exp Brain Res 121:391-400.

Ogawa S, Tank DW, Menon R, Ellermann JM, Kim SG, Merkle H, Ugurbil K (1992) Intrinsic signal changes accompanying sensory stimulation: functional brain mapping with magnetic resonance imaging. Proc Natl Acad Sci U S A 89:5951-5955. 
Ojemann JG, Akbudak E, Snyder AZ, McKinstry RC, Raichle ME, Conturo TE (1997) Anatomic localization and quantitative analysis of gradient refocused echo-planar fMRI susceptibility artifacts. Neuroimage 6:156-167.

Oldfield RC (1971) The assessment and analysis of handedness: the Edinburgh inventory. Neuropsychologia 9:97-113.

Ollinger JM, Shulman GL, Corbetta M (2001a) Separating processes within a trial in event-related functional MRI. Neuroimage 13:210-217.

Ollinger JM, Corbetta M, Shulman GL (2001b) Separating processes within a trial in event-related functional MRI. Neuroimage 13:218-229.

Polli FE, Barton JJ, Cain MS, Thakkar KN, Rauch SL, Manoach DS (2005) Rostral and dorsal anterior cingulate cortex make dissociable contributions during antisaccade error commission. Proc Natl Acad Sci U S A 102:15700-15705.

Rosenberg DR, Sweeney JA, Gillen JS, Kim J, Varanelli MJ, O’Hearn KM, Erb PA, Davis D, Thulborn KR (1997) Magnetic resonance imaging of children without sedation: preparation with simulation. J Am Acad Child Adolesc Psychiatry 36:853-859.

Sakai K (2008) Task set and prefrontal cortex. Annu Rev Neurosci 31:219_ 245.

Snyder AZ (1996) Difference image versus ratio image error function forms in PET-PET realignment. In: Quantification of brain function using PET (Bayley D, Jones T, eds), pp 131-137. San Diego: Academic.

Talairach J, Tournoux P (1988) Co-planar stereotaxic atlas of the human brain. New York: Thieme Medical.

The Psychological Corporation (1999) Wechsler Abbreviated Scale of Intelligence (WASI) manual. San Antonio, TX: The Psychological Corporation.
Tregellas JR, Davalos DB, Rojas DC (2006) Effect of task difficulty on the functional anatomy of temporal processing. Neuroimage 32:307-315.

Velanova K, Jacoby LL, Wheeler ME, McAvoy MP, Petersen SE, Buckner RL (2003) Functional-anatomic correlates of sustained and transient processing components engaged during controlled retrieval. J Neurosci 23:8460-8470.

Velanova K, Wheeler ME, Luna B (2008) Maturational changes in anterior cingulate and frontoparietal recruitment support the development of error processing and inhibitory control. Cereb Cortex 18:2505-2522.

Visscher KM, Miezin FM, Kelly JE, Buckner RL, Donaldson DI, McAvoy MP, Bhalodia VM, Petersen SE (2003) Mixed blocked/event-related designs separate transient and sustained activity in fMRI. Neuroimage 19:16941708.

Woodruff CC, Uncapher MR, Rugg MD (2006) Neural correlates of differential retrieval orientation: sustained and item-related components. Neuropsychologia 44:3000-3010.

Worsley KJ, Friston KJ (1995) Analysis of fMRI time-series revisitedagain. Neuroimage 2:173-181.

Xue G, Aron AR, Poldrack RA (2008) Common neural substrates for inhibition of spoken and manual responses. Cereb Cortex 18:1923-1932.

Zarahn E, Aguirre GK, D’Esposito M (1997) Empirical analyses of BOLD fMRI statistics. I. Spatially unsmoothed data collected under nullhypothesis conditions. Neuroimage 5:179-197.

Zheng D, Oka T, Bokura H, Yamaguchi S (2008) The key locus of common response inhibition network for no-go and stop signals. J Cogn Neurosci 20:1434-1442. 